Improving Perinatal Care Through Theory Application

By: Denise Côté-Arsenault and Lori Johnson Hubbard

Côté-Arsenault, D. Hubbard, L.J. Improving Perinatal Care Through Theory Application. MCN, The American Journal of Maternal/Child Nursing, 44(6) 345-350. DOI:

10.1097/NMC.0000000000000564

Made available courtesy of Lippincott, Williams \& Wilkins:

http://dx.doi.org/10.1097/NMC.0000000000000564

***;C 2019 Wolters Kluwer Health, Inc. Reprinted with permission. No further

reproduction is authorized without written permission from Wolters Kluwer/Lippincott, Williams \& Wilkins. This version of the document is not the version of record. Figures and/or pictures may be missing from this format of the document. ***

\begin{abstract}
:
Theories from various perspectives can increase nurses' understanding of maternal behaviors throughout the developmental process of pregnancy and postpartum. The purpose of this article is to present relevant theories that maternity nurses will likely find useful, and to demonstrate their applicability through an unfolding exemplar case. Nurses can provide improved and more sensitive care when guided by psychosocial theories such as Rubin's tasks of pregnancy, prenatal attachment, pregnancy as a liminal phase, and the grief work in postpartum as proposed by Mercer. Use of relevant theories can promote holistic nursing care, increase critical thinking, and improve nursing responsiveness to unique family situations such as pregnancy after loss and premature birth.
\end{abstract}

Keywords: Grief | Pregnancy | Prenatal attachment | Theory

\title{
Article:
}

Caring for women and families during the perinatal period is a complex endeavor as it involves several aspects of human experience; it is a biopsychosocial developmental process (Rubin, 1984) as well as a spiritual one (McEwen, 2014). The woman initially feels physical changes in her own body and perceives the hidden embryo or fetus as part of herself while the father has only a mental sense that profound changes are occurring (Genesoni \& Tallandini, 2009). Across the 9 months of fetal development, the expectant mother and her spouse or partner begin to recognize changes in themselves, each other, as well as their social relationships as they are now seen as soon-to-be parents (Slade, Cohen, Sadler, \& Miller, 2009). Birth of the newborn ushers in postpartum emotional and social adaptations for the new family.

Excellent nursing care requires attention to the physical body and its changing systems, as well as knowledge about expected psychosocial changes and how to support women and families as they navigate many changes. Theories (including conceptual frameworks) from various 
disciplines such as psychology, nursing, and anthropology can increase nurses' understanding of what the mother and family members are experiencing. Knowledge of theoretical bases for nursing care extends our expectations for normal adaptations to pregnancy and postpartum and expands our repertoire of holistic interventions (McEwen, 2014). Conceptual frameworks can be very similar in structure and use as theories. The purpose of this article is to introduce and apply several theories relevant to perinatal nursing care through an unfolding exemplar case.

\section{Use of Theory}

Use of theories to guide nursing practice is not a new idea. Nurses use theories originating in the natural and social sciences throughout their practice, but many are not aware of theory utilization in making decisions (McEwen, 2014). For example, infection control theories guide handwashing to reduce infection rates. Theories guide our assessments and actions because they become lenses through which we see the world. One example is the transition from exclusion of fathers during labor and birth to family-centeredness. Now that practice, societal expectations, and clinical environments have evolved, it is difficult not to view care through a family-centered lens. Once nurses' views are broadened, they are forever changed.

One of many suggested definitions of theory is that it is a set of assumptions or principles concerning logically interrelated concepts developed to describe, explain, or predict phenomena or behaviors (Goodson, 2011). The most helpful theories are relevant, meaningful, and understandable to those considering their use. Nurses who work with families during the perinatal period need to have a repertoire of theories to apply as they care for the mother, fetus, newborn, and other family members. Theories about pregnancy and becoming a parent provide the knowledge base for the maternity nursing specialty, differentiating and guiding the thought processes of nurses providing care across the childbearing continuum. Practice informs theory and theory informs practice; they are integrally connected and reciprocal (McEwen, 2014). Practice guided by theory, as presented here, will be improved, more responsive, and will lead to more holistic family care.

\section{Exemplar Case_-Part 1}

Ashley was excited to hear a fetal heartbeat at her 10th and 14th week prenatal care visits during her first pregnancy. Other than a few weeks of nausea and fatigue, she experienced a first trimester free of complications. During a routine anatomy scan at 18 weeks, Ashley and her husband Jason were devastated when no fetal heartbeat was detected. After an induction and birth, they created brief memories with their tiny son. The following weeks were filled with grief and disorientation. The couple found a local support group where they slowly began to work through their feelings of grief and loss with other bereaved parents.

Following pregnancy announcements from several close friends and family members and a continued longing to grow their family, Ashley and Jason decided to try again for another child. Approximately 10 months after the loss, Ashley was pregnant again.

Ashley and Jason shared news of the current pregnancy with their parents but asked them not to say anything until they felt sure the pregnancy was "OK." Because there was no known reason for the loss of her first pregnancy, Ashley called the nurse line frequently with questions about 
aches and pains, and potential hazards. She checked for blood every time she used the toilet. She found comfort in her morning sickness because it meant that she was still pregnant. Prior to their upcoming anatomy scan, both parents were very anxious. They recalled their painful experience learning of their first baby's demise during the ultrasound. Although they had heard a fetal heartbeat multiple times, both Ashley and Jason cried tears of relief and joy when a 20week ultrasound revealed a strong heartbeat. No abnormalities were found, and they were told that their healthy baby was a girl.

\section{Pregnancy as a Rite of Passage and Liminal Phase}

Although pregnancy is easily seen as a physical state by nurses, it is also an integral and momentous part of our sociocultural lives. Viewing pregnancy and childbearing as a social rite of passage broadens one's thinking beyond the usual health and medical models of care. Turning to the social sciences, particularly anthropology, it becomes clear that pregnancy can affect the social status of the mother and the father, affecting the broader family and community.

Pregnancy is generally not a solitary event or process; it is a major life event that is acknowledged and celebrated by many and is a transition from "woman" to "woman-mother." Arnold van Gennep, an early 20th century French anthropologist, was the first to use the phrase "rites of passage" (1960). He used this in reference to moving from one social status to another and identified three phases of this passage: separation, transition, and incorporation. In the case of pregnancy in our dominant culture, the rite of separation occurs when the pregnancy is announced socially. The transition, or liminal phase, is the longest and most challenging part. It is marked by regular prenatal visits, hormonal shifts, increasing girth, prenatal classes, and baby showers. Finally, the rite of incorporation generally occurs for the mother when she brings her baby home and introduces the baby to her family, friends, co-workers, and neighbors (CôtéArsenault, Brody, \& Dombeck, 2009). She is then seen as "woman-mother." Hence, the word mother is used throughout this article. Each of these visible or social events serves to transform how the parents are seen by others in their social sphere. Therefore, after bringing the newborn home, the parents' new social status changes how they are viewed and treated, affects the roles and relationships they have in society, and alters the social norms that influence their behavior (Côté-Arsenault et al.; van Gennep).

The liminal or transition phase was deemed by the anthropologist Victor Turner (1987) as a precarious time that is disruptive to previous norms. Turner sees individuals as "betwixt and between" two social statuses in liminality, a place of uncertainty. This is the case in pregnancy, most notably in a first pregnancy. Rituals are known to cushion disruption and ease the transition. Rituals such as prenatal care visits and baby showers are readily acknowledged as steps toward motherhood by members of society. Individuals and families sometimes create their own ways to mark off weeks of pregnancy such as updates on social media with photographs of the mother's growing belly. Rituals or ceremonies in the liminal phase of pregnancy may vary by culture. Indeed, in cultures such as Arabic Muslim or Eastern European announcement of pregnancy or naming of the baby during pregnancy is avoided until the pregnancy outcome is known (Callister, 2014).

\section{Application}


In the exemplar case, Ashley and her husband entered the transition into their first pregnancy when they started prenatal visits. However, they were stuck in liminality when that pregnancy ended in fetal demise. They did not bring that baby home and thus had no public rite of incorporation. Socially they were not seen as parents. So, it is not surprising that when they became pregnant again they felt uncertain, anxious, and worried. They tentatively announced the pregnancy to only a few family members, which is more often the case in pregnancy after loss (Côté-Arsenault et al., 2009; Côté-Arsenault \& O'Leary, 2016).

Nurses who understand rites of passage in pregnancy can use their cultural and societal lens to provide a more finely tuned level of care. Nurses caring for families with a history of pregnancy loss or other complicated pregnancies can assess the extent of the mother's announcement of the pregnancy to others, along with her anxiety or tentativeness. Understanding that pregnancy is a liminal phase explains and normalizes the worry over a current pregnancy when a prior pregnancy did not follow the anticipated course (Côté-Arsenault \& O'Leary, 2016). Providing rituals through asking routine questions, giving ongoing support, anticipatory guidance, and recognition of anxiety can serve to buffer the liminality of pregnancy.

\section{Rubin's Maternal Tasks of Pregnancy}

During the 1960-70s, Reva Rubin, a nurse researcher and theorist, studied women throughout pregnancy and postpartum periods. Nurses are most familiar with Rubin's theory of Maternal Role Attainment in the postpartum period as the mother moves through the stages of taking in, taking hold, and letting go. However, nurses involved in prenatal care will find Rubin's concept of a developing Maternal Identity an invaluable lens through which to view developmental changes and behaviors. Maternal Identity is developed through a pregnant woman undertaking four tasks of pregnancy: 1) ensuring safe passage of self and baby, 2) ensuring social acceptance of self and baby, 3) binding-in to the baby, and 4) giving of self (Rubin, 1984).

Safe passage, the first task a woman undertakes early in pregnancy, involves prenatal care and concern about the health and safety of herself and the fetus. A woman may alter her diet or environment to avoid anything that could cause harm to her unborn baby while also taking prenatal vitamins to enhance her health (Rubin, 1984). Safe passage concerns emerge again in the third trimester as thoughts and fears about labor and birth predominate. Social acceptance is sought through announcing the pregnancy to family and friends in the hopes that they will respond with excitement and approval. Another aspect of social acceptance occurs when the woman considers how the addition of a baby will change the fabric of her social groups and interactions (Rubin). Binding-in begins when the idea of a fantasized, theoretical baby gives way to the awareness of the child within. It increases sharply with quickening in the second trimester. It is associated with an emotional attachment to the child as an entity separate from herself (Rubin).

Giving of self is the bookend of a pregnancy that started with the safe passage task. In this complex task, the mother makes lifestyle choices that will extend beyond the pregnancy. She may cut back on extraneous commitments in preparation to care for her infant (Rubin, 1984). 
This may be more difficult for women today with juggling multiple priorities than in Rubin's time when women were less likely to have professional priorities outside the home.

The theoretical tasks occur in a general order, but they may overlap. An expectant mother may get "stuck" on a particular task or show no sign of developing a maternal identity; these should serve as a red flag to the nurse. Awareness of these tasks can guide nurses' theorizing and lead to more individualized and effective care.

\section{Prenatal Attachment}

Rubin's recognition of binding-in is consistent with the concept of prenatal attachment (Cranley, 1981). The notion of attachment was introduced by John Bowlby, a psychoanalyst in the 1970s whose focus was on the child's attachment to the caregiver. In contrast, the focus here is on the bonds that are forming between the mother to her inner baby and the father's emotional ties to his fantasized future child.

Maternal-fetal attachment was primarily conceptualized by nurses such as Rubin (1961) and Cranley (1981). Later, conceptions of prenatal attachment have focused on the mother's and father's psychological bond to the unborn child that is visible in parental behaviors (Brandon, Pitts, Denton, Stringer, \& Evans, 2009; Condon, 1985; Klaus \& Kennell, 1976). Prenatal attachment generally increases across the pregnancy, facilitated by the realism of hearing the fetal heartbeat, seeing the baby on ultrasound, and feeling fetal movements. Mothers have higher levels of attachment than fathers until after birth when both parents see, hold, and care for their baby (Brandon et al.). Nurses can note signs of prenatal attachment through assessing the way expectant mothers speak about their baby, call the baby by name, or wrap their hand protectively over their protruding belly. An indicator that prenatal attachment has not occurred or is being disrupted may be observed in a woman showing a lack of interest in her unborn baby.

\section{Application}

In both Ashley's first and second pregnancy, she exhibited the task of safe passage by beginning prenatal care. Without awareness of safe passage as a naturally occurring stage intensified by prior loss, nurses may become frustrated with Ashley's calls or more frequent office visits. Although Ashley was quite careful about her diet in her first pregnancy, she decided to eliminate coffee during her second pregnancy "just to be safe" (Côté-Arsenault, 1995).

The task of social acceptance has changed a great deal in recent years due to popularity of social media. Mothers may feel an urgency to create frequent posts to social media sites or have a gender reveal party that may conflict with aspects of the pregnancy she and her partner originally thought they would keep personal and intimate. However, social networks may serve as a source of encouragement. Haddrill, Jones, Anumba, and Mitchell acknowledge a "personal" pregnancy where a woman seeks support from her immediate social circle and a "public" pregnancy fueling socially accepted routines such as initiating and receiving prenatal care (2018, p. 222). Nurses should assess the availability and level of activity of immediate family as well as the woman's extended layers of social support to determine navigation through 
the task of social acceptance. Support persons should be made aware of symptoms of risk factors in pregnancy, specifically mental health disorders, because women often confide in their immediate support network prior to seeking professional services (Fonseca \& Canavarro, 2017).

The development of prenatal attachment and binding-in can be assessed by the expectant mother's reactions during ultrasounds and fetal heart rate assessments where nurses can gain insight into the mother's degree of binding-in. Education about fetal kick counts could also include information about binding-in and strengthening prenatal attachment. Providing ultrasound photos or videos can promote prenatal attachment for the spouse or partner. Nurses should watch for delayed binding-in when a mother has had a previous loss or has a complicated pregnancy. Her reality is that not all pregnancies end with the birth of a healthy baby so self-protection, called emotional cushioning, is common (Côté-Arsenault \& Donato, 2011). Nurses should assure these mothers that fear and anxiety are normal and may be manifested as muted prenatal attachment.

Exemplar Case-Part 2

At 30 weeks, Ashley began to experience regular contractions; she and her husband were quite distressed. Upon arrival at the hospital, her cervix was dilated $4 \mathrm{~cm}$. Despite medical efforts to stop labor, Ashley birthed a 1,250 gram female the next day. The premature neonate was transferred to a Neonatal Intensive Care Unit (NICU) at a hospital 45 minutes away. The baby's first week was complicated by respiratory distress syndrome, yet Ashley was able to begin skinto-skin time 5 days after the birth. She had not been able to attend childbirth and breastfeeding classes prior to giving birth. Ashley expressed sadness and often cried at the baby's bedside. She often asked the nursing staff what she did to cause the premature birth.

\section{Grief Work Postpartum}

It is not uncommon for women who give birth preterm to grieve the perfect pregnancy and birth. Ramona Mercer, a student of Rubin, viewed the psychological work of adjusting from being pregnant with an internal child to the reality of a newborn in arms as grief work (1981). While a woman is pregnant, she envisions herself as a mother and imagines what her newborn will be like; these fantasies help her to picture herself in the role of mother. After birth, the new mother must reconcile her hopes and dreams for self, labor and birth, and baby with real events (Rubin, 1984). According to Mercer "the mother must reconcile the baby she had with her fantasy baby of pregnancy" (p. 342). Acceptance of the outer baby is a requisite psychological adaptation that allows the mother to care for and be responsive to the infant, with greater incongruencies requiring more grief work.

\section{Application}

Ashley and her husband must grieve for the normal birth and homecoming they had imagined. Although they have a beautiful outer baby, their small daughter needs NICU staff care. Nurses could tell these parents that it is normal and okay to grieve and guide them by providing words to name their emotions. 
Nurses can play a major role in facilitating mothers' integration of their expectations with reality through asking a mother if she would like to talk about the labor and birth experience. The act of telling the birth story may help some mothers close the gap between the imagined scenario and the actual one (Carson et al., 2017). The nurse should be sensitive to any conflicts or concerns expressed and respond in a way that leaves the discussion open rather than giving quick answers or solutions. Listening carefully will also help the mother move toward acceptance of reality and emotional attachment to her baby. It may also prove helpful to tell a mother that it is normal to feel sad about leaving pregnancy behind prematurely, but then facilitate her focus on the newborn she has in her arms or in the NICU (if that is the case).

A preterm birth requiring nursing care of a newborn in the NICU, where preparation for motherhood ended abruptly, is a rich opportunity for recognition of theory to guide best practices in nursing. The mother may not have yet moved through all of Rubin's tasks of pregnancy. Preterm birth is a crisis; safe passage was not fully achieved. Nurses should recognize their role in supporting the completion of social acceptance through family visitation, webcam viewings, and possibly facilitating baby showers. In the exemplar case, Ashley may grapple with the task of giving of self if she was ambivalent about her feeding plans but is now being encouraged to supply breast milk to her preterm neonate. She is having to "give of herself" and needs education and support from the staff. Nurses can give lactation support and praise her efforts to provide any amount of breastmilk for her infant.

The rite of incorporation of taking the baby home is often delayed with a NICU stay. Being discharged with empty arms is often traumatic; she is a mother but cannot yet introduce her newborn to people in her social circle. Recognizing this emotional event, rituals such as carrying a teddy bear or bouquet of flowers could fill a mother's arms; looking at a photo of her baby en route home might reinforce baby's reality and ease the transition. The parents may also be comforted by regular updates on the baby as well as opportunities for parent caregiving and skinto-skin care in the NICU (Feeley, Genest, Niela-Vilén, Charbonneau, \& Axelin, 2016).

\section{Discussion}

This article presents theories relevant to maternity nursing care and applies them to an unfolding exemplar case. Although the case presented two high-risk situations - pregnancy after loss and premature birth - these theories are applicable to all childbearing families. They may help nurses consider pregnancy as a unique developmental state with predictable changes and desired outcomes. The range of perinatal cases is endless, but these theories provide guidance about expected behaviors and how to explain or understand unexpected ones. Prepared with this understanding, nurses become more sensitive and responsive to looking below the surface and consider seeking more information, if needed.

Integrating theory-based care from a variety of disciplines into practice has the potential to facilitate more holistic care where psychological and cultural views are considered. It should be noted, however, that additional research is needed to explore the impact of socioeconomic, environmental, age, and racial variables on the way pregnancy-related theories are expressed. For example, Fouquier (2013), in a state of the science analysis of maternal role attainment in African-American women, noted that most of theory development and research has focused on 
middle-income, Caucasian women. Insight into theory expression in diverse populations will give nurses additional tools for personalizing care for women transitioning to motherhood including prenatal education and increased commitment with health coaching and recommendations (Ross, 2012) and development of interventions for NICU mothers (Hall, Kronborg, Aagaard, \& Brinchmann, 2013). A lifespan approach to understanding maternal identity development can guide nursing care of pregnant adolescents (Macintosh \& Callister, 2015) as well as an awareness of the dual liminality of being within pregnancy and not yet an adult.

\section{Implications for Practice}

As women move from pregnancy to postpartum, they may be unprepared to encounter feelings of grief. A common postpartum example is the new mother who says she feels so empty without her baby inside. A new theoretical lens allows that statement to be interpreted as grief work. The nurse could possibly say, "I hear many mothers say how they miss being pregnant and feeling baby's movement inside. Can you see how your newborn's movements on the outside are the same as movements you felt while pregnant?" This response acknowledges the mother's grief as normal, confirming that the outer baby replaces the inner baby, and encourages continued maternal attachment to the reality of the newborn.

When theories are integral to nurses' thinking, maternal behaviors will be assessed as consistent or inconsistent with theoretical expectations. When inconsistencies are noted, the nurse will seek further understanding of what is going on with an individual or family. A case in point would be a woman who misses numerous appointments or delays prenatal care. This behavior could be indicative of a cultural variation, disinterest in seeking safe passage, or the result of transportation issues. Through a theory lens, the nurse broadens her assessment to reveal root causes for behavior and direction for intervention. Provision of additional supports and information about resources could be done with new insights.

Becoming more familiar and comfortable with new theories does require some effort on the nurse's part. The first step is to select a theory of interest and then locate additional reading about the theory and its use. Assistance can be found in clinical nurse specialists, nurse educators, or librarians. Within the clinical setting, a discussion group can be initiated to study the theory, examine the range of cases seen on a clinical unit, and explore the application of theory to nursing care. Practice changes can occur individually, within a unit, or through policy change.

The use of theories related to nursing begins with appreciating what theory-guided practice offers and ensuring one's familiarity with relevant theories. Staff nurses may not be cognizant of their current use of theories in their critical thinking and care planning. The exemplar case presented here may bring related theories to conscious thought. Theory use can facilitate greater understanding of normal responses to pregnancy, increase recognition of variations in behavior, and result in a wider repertoire of responses. Holistic care, a hallmark of nursing, will be enhanced through the application of theories in clinical practice.

\section{Clinical Implications}


- Nurses who work with women and their families during childbirth need a repertoire of theories to apply as they provide care to the mother, fetus, newborn, and other family members.

- When nurses' thinking is theory-based, maternal behaviors can be assessed for consistency with what is expected based on each theory.

- Integrating theory-based care into practice, particularly from a variety of disciplines, will lead to more holistic care where psychological and cultural perspectives are considered.

\section{References}

Brandon A. R., Pitts S., Denton W. H., Stringer C. A., Evans H. M. (2009). A history of the theory of prenatal attachment. Journal of Prenatal \& Perinatal Psychology and Health, 23(4), 201-222.

Callister L. C. (2014). Integrating cultural beliefs and practices when caring for childbearing women and families. In K. R. Simpson \& P. A. Creehan (Eds.), AWHONN's perinatal nursing (4th ed., pp. 41-70). Philadelphia, PA: Wolters Kluwer/Lippincott Williams \& Wilkins.

Carson A., Chabot C., Greyson D., Shannon K., Duff P., Shoveller J. (2017). A narrative analysis of the birth stories of early-age mothers. Sociology of Health \& Illness, 39(6), 816-831. doi:10.1111/1467-9566.12518

Condon J. T. (1985). The parental-foetal relationship_A comparison of male \& female expectant parents. Journal of Psychosomatic Obstetrics \& Gynecology, 4(4), 271-284. doi:10.3109/01674828509016729

Côté-Arsenault D. (1995). Tasks of pregnancy and anxiety in pregnancy after perinatal loss. Dissertation Abstracts International, 56(12), 6669.

Côté-Arsenault D., Brody D., Dombeck M. T. (2009). Pregnancy as a rite of passage: Liminality, rituals \& communitas. Journal of Prenatal \& Perinatal Psychology and Health, 24(2), 69-87.

Côté-Arsenault D., Donato K. (2011). Emotional cushioning in pregnancy after perinatal loss. Journal of Reproductive and Infant Psychology, 29(1), 81-92. doi:10.1080/02646838.2010.513115

Côté-Arsenault D., O'Leary J. (2016). Understanding the experience of pregnancy subsequent to perinatal loss. In B. Black, P. Wright, \& R. Limbo (Eds.), Perinatal and pediatric bereavement in nursing and other health professions (pp. 159-181). New York, NY: Springer Publishing.

Cranley M. S. (1981). Development of a tool for the measurement of maternal attachment during pregnancy. Nursing Research, 30(5), 281-284. doi:10.1097/00006199-198109000-00008

Feeley N., Genest C., Niela-Vilén H., Charbonneau L., Axelin A. (2016). Parents and nurses balancing parent-infant closeness and separation: A qualitative study of NICU nurses' perceptions. BMC Pediatrics, 16, 134. doi:10.1186/s12887-016-0663-1 
Fonseca A., Canavarro M. C. (2017). Women's intentions of informal and formal help-seeking for mental health problems during the perinatal period: The role of perceived encouragement from the partner. Midwifery, 50, 78-85. doi:10.1016/j.midw.2017.04.001

Fouquier K. F. (2013). State of the science: Does the theory of maternal role attainment apply to African American motherhood? Journal of Midwifery \& Women's Health, 58(2), 203-210. doi:10.1111/j.1542-2011.2012.00206.x

Genesoni L., Tallandini M. A. (2009). Men's psychological transition to fatherhood: An analysis of the literature, 1989-2008. Birth, 36(4), 305-318.

Goodson P. (2011). Theory as practice. In J. B. Butts \& K. L. Rich (Eds.), Philosophies and theories for advanced nursing practice (pp. 71-87). Sudbury, MA: Jones \& Bartlett.

Haddrill R., Jones G. L., Anumba D., Mitchell C. (2018). A tale of two pregnancies: A critical interpretive synthesis of women's perceptions about delayed initiation of antenatal care. Women and Birth, 31(3), 220-231. doi:10.1016/j.wombi.2017.09.017

Hall E. O. C., Kronborg H., Aagaard H., Brinchmann B. S. (2013). The journey towards motherhood after a very preterm birth: Mothers' experiences in hospital and after homecoming. Journal of Neonatal Nursing, 19, 109-113. doi:10.1016/j.jnn.2012.08.002

Klaus M., Kennell J. (1976). Maternal-infant bonding. St. Louis, MO: Mosby.

Macintosh J., Callister L. C. (2015). Discovering self: Childbearing adolescents' maternal identity. MCN. The American Journal of Maternal Child Nursing, 40(4), 243-248.

doi:10.1097/NMC.0000000000000143

McEwen M. (2014). Application of theory in nursing practice. In M. McEwen \& E. M. Wills (Eds.), Theoretical basis for nursing (4th ed., pp. 412-429). Philadelphia, PA: Wolters Kluwer.

Mercer R. T. (1981). The nurse and maternal tasks of early postpartum. MCN. The American Journal of Maternal Child Nursing, 6(5), 341-345.

Ross E. (2012). Maternal-fetal attachment and engagement with antenatal advice. British Journal of Midwifery, 20(8), 566-575. doi:10.12968/bjom.2012.20.8.566

Rubin R. (1961). Basic maternal behavior. Nursing Outlook, 9, 683-686. PMID: 14494924

Rubin R. (1984). Maternal identity and the maternal experience. New York, NY: Springer Publishing Company.

Slade A., Cohen L. J., Sadler L. S., Miller M. (2009). The psychology and psychopathology of pregnancy. Reorganization and transformation. In C. H. Zeanah (Ed.), Handbook of infant mental health (pp. 22-39). New York, NY: Guilford Press. 
Turner V. (1987). Betwixt and between: The liminal period in rites of passage. In L. C. Mahdi, S. Foster, \& M. Little (Eds.), Betwixt and between: Patterns of and feminine initiation (pp. 3-19). LaSalle, IL: Open Court. (Original published 1967.)

van Gennep A. (1960). The rites of passage. (M. B. Vizedom and G. L. Caffee, Trans.) Chicago, IL: The University of Chicago Press. (Original work published 1908.) 